\title{
Differential Pigment Accumulation In Carrot Leaves And Roots During Two Growing Periods
}

Florent Perrin, ${ }^{\dagger \dagger}$ Marwa Brahem, ${ }^{\dagger \dagger}$ Cécile Dubois-Laurent, ${ }^{\dagger}$ Sébastien Huet, ${ }^{\ddagger}$ Matthieu Jourdan, ${ }^{\dagger \ddagger}$ Emmanuel Geoffriau, ${ }^{\ddagger}$ Didier Peltier, ${ }^{\dagger}$ and Séverine Gagné ${ }^{\dagger *}$

${ }^{\dagger}$ Université d'Angers, Institut de Recherche en Horticulture et Semences - UMR 1345, SFR 4207 QUASAV, Angers, France

${ }^{\ddagger}$ Agrocampus Ouest, Institut de Recherche en Horticulture et Semences - UMR 1345, SFR 4207 QUASAV, Angers, France

*Corresponding author: Séverine Gagné (severine.gagne@ univ-angers.fr), TEL 33.2.41.22.57.30, FAX 33.2.41.22.55.15. 


\section{SUPPORTING INFORMATION}

Supporting Information Table 1: Primer sequences used for gene expression analysis

\begin{tabular}{|c|c|c|c|}
\hline Gene & Forward primer (from 5' to 3') & Reverse primer (from 5' to 3') & $\begin{array}{l}\text { Accession } \\
\text { number }\end{array}$ \\
\hline$E F 1 \alpha$ & GGAACCTCTCAGGCTGATTGTG & TTGGAGATACCAGCTTCAAAACC & D12709 \\
\hline Actin & CCACACGGTGCCAATTTATG & GATCACGGCCAGCAAGGT & $\mathrm{X} 17526.1$ \\
\hline Tubulin $\beta$ & GCCGAGAGCAACATGAATGAC & CCTGTTCATCTTCGTAGTAATCTTCCT & $\mathrm{X} 16608.1$ \\
\hline GGPPS & GCTACTGAGACCAGCGGAGT & CCCATAACAACCGAACCTT & DQ192184 \\
\hline PSY1 & TTGGGCAATCTATGTGTGGTGTAG & GCCTTGGGCGTGATATGG & DQ192186 \\
\hline PSY2 & TCAGTTGCTATGTCCTGGATTGTT & CCTCTCGGCAGTCTCAA & DQ192187 \\
\hline$P D S$ & TAACATGGCCTGAGAAGATCAAAGT & CACGTAGGCTTGTCCACCAA & DQ222429 \\
\hline$L C Y B 1$ & AGTGGGAATTGGCGGTACAG & GCAGCTAGAGTTCTTGCTACCATATATC & DQ192190 \\
\hline$L C Y E$ & CATTCCATGCAGGCTTGCTA & AACCTCATACTGCAAAAGTT & DQ192192 \\
\hline$Z E P$ & CGGAGAAATTTGTTTGGATACACAGAA & CAATATCTGCAGGCACAAAATCTGC & DQ192197 \\
\hline NCED1 & CATTCCGGGATAGCGCGGCTC & CCAACCCCGCATTCGCAACC & DQ192200.1 \\
\hline NCED2 & GGATCAAGTGAATCTGGAGGCAGGG & CAGCAATGGCCAAATAAGCAAACTGG & DQ192201.1 \\
\hline NCED3 & CCAGTCCAGCACCACCTCCCT & TGGACCATGCCATCCCCGTCA & DQ102202.1 \\
\hline$A B A 2$ & ACGCCAATGGCTTTTGGCCACT & TTCGTGGTCAGCTCCACGCC & VCDC17045 \\
\hline
\end{tabular}


Supporting Information Figure 1: Changes in climatic parameters during the two growing periods. Temperature (A) and global radiation (B) during the first growing period. Temperature (C) and global radiation (D) during the second growing period. The dotted lines correspond to the period mean of each parameter.
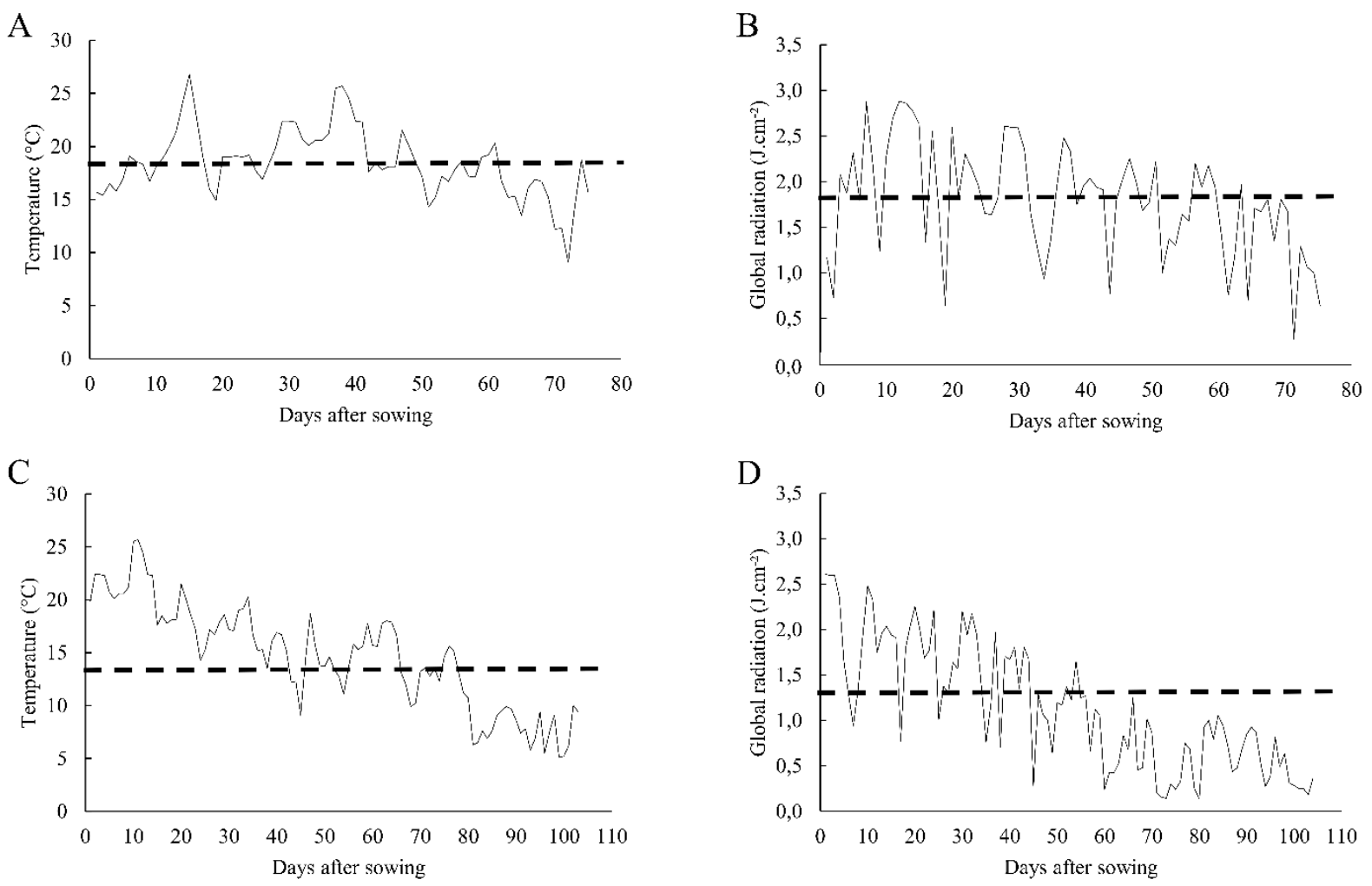
Supporting Information Figure 2: Principal component analysis factor map based on pigment compounds in carrot leaves. (A) Individual contribution of each variable to the first and the second dimensions. Sample distribution is shown by (B) growing period and (C) by genotype. Each dot is the mean of three biological replications ( $n=3)$. GP 1: growing period 1, GP 2: growing period 2, chla: chlorophyll a, chlb: chlorophyll b, X9bcarotene: 9 -cis- $\beta$-carotene, a.carotene: $\alpha$-carotene, b.carotene: $\beta$-carotene.
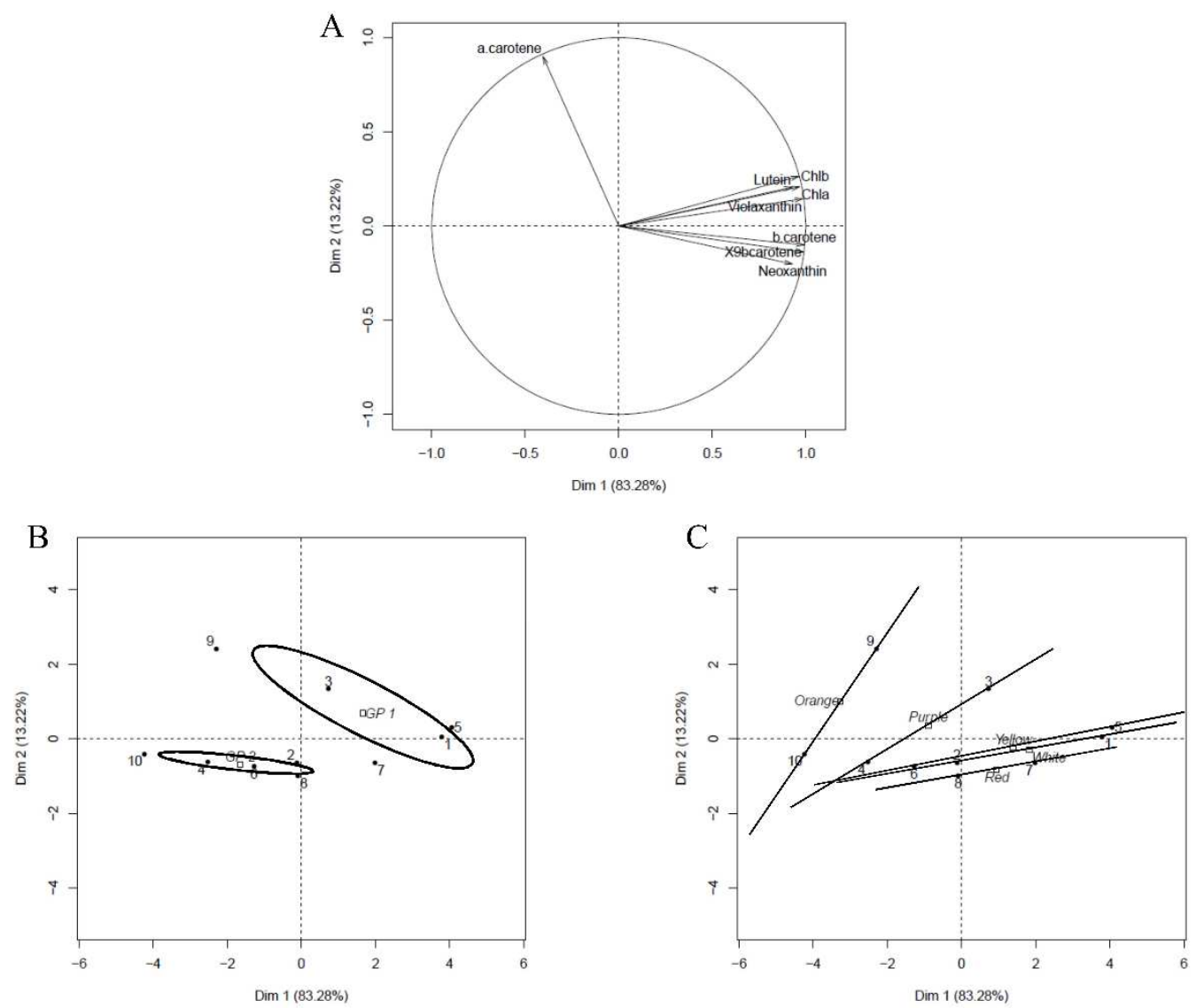
Supporting Information Figure 3: Principal component analysis factor map based on carotenoid contents in carrot roots. (A) Individual contribution of each variable to the first and the second dimensions. Sample distribution is shown by (B) growing period and (C) by genotype. Each dot is the mean of three biological replications $(n=3)$. GP 1: growing period 1, GP 2: growing period 2, a.carotene: $\alpha$-carotene, b.carotene: $\beta$-carotene.
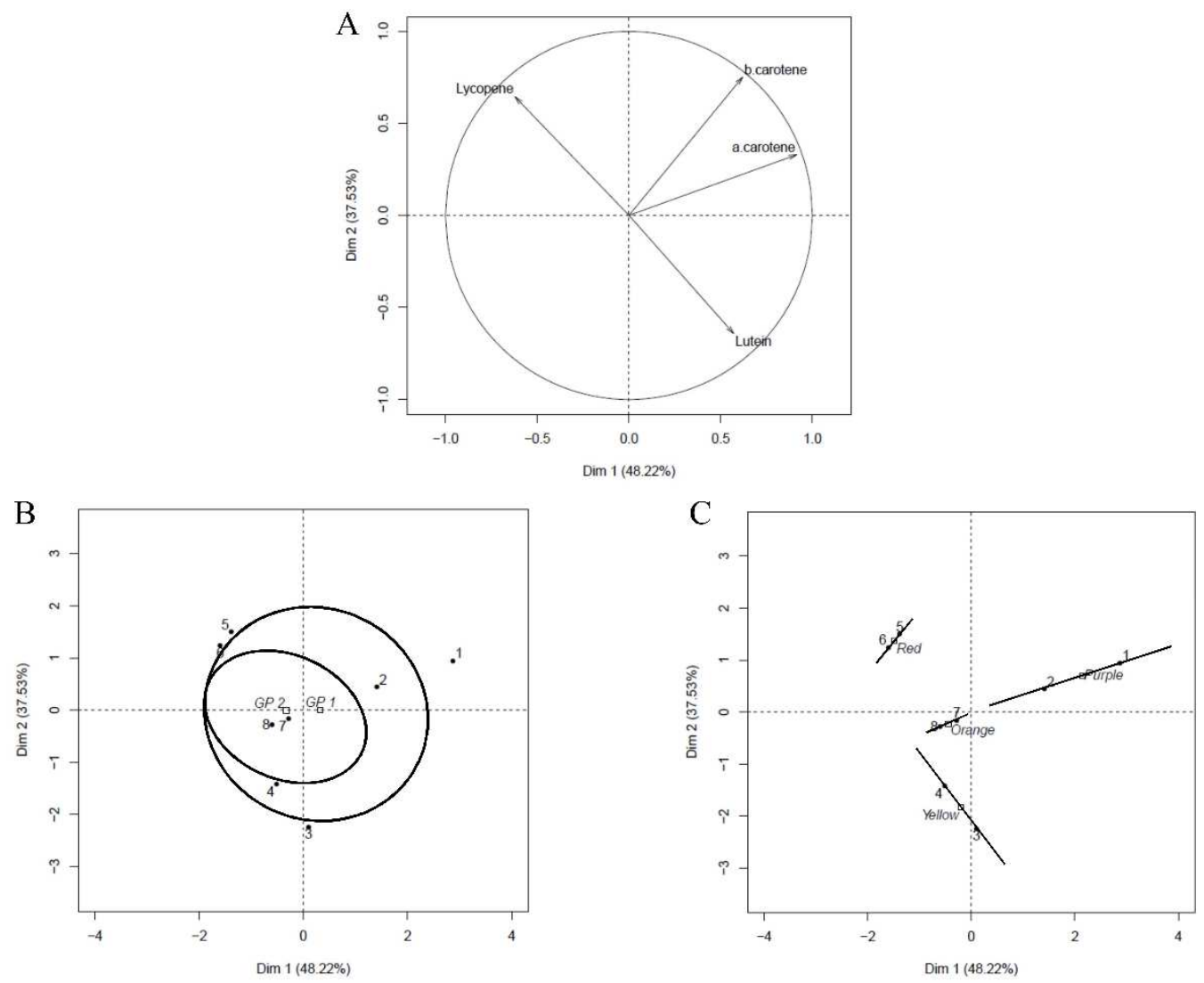